\title{
A Cross-Sectional Study of Clinical and Immunological Profile of SLE Patients from a Tertiary Care Center in the Sub Himalayan Region of Northern India
}

\author{
Varun Jaswal $^{1}$, Jitender K Mokta ${ }^{2}$, Vivek Chauhan ${ }^{3}$, Vandna Raghuvanshi ${ }^{4}$, \\ Vineet Kumar', Aarti Dhatwalia ${ }^{6}$ \\ ${ }^{1}$ Medical Officer (Medicine), Pt. JLNGMC Chamba, Himachal Pradesh. \\ ${ }^{2}$ Professor, Department of Medicine, IGMC Shimla, Himachal Pradesh. \\ ${ }^{3}$ Assistant Professor, Department of Medicine, IGMC Shimla, Himachal Pradesh. \\ ${ }^{4}$ Assistant Professor, Department of Radiodiagnosis, Pt. JLNGMC Chamba, Himachal Pradesh. \\ ${ }^{5}$ Senior Resident, Department of Community Medicine, IGMC Shimla, Himachal Pradesh \\ ${ }^{6}$ Medical Officer (Pathologist), CMO Office Shimla, Himachal Pradesh.
}

Corresponding Author: Aarti Dhatwalia

\begin{abstract}
Introduction: Systemic Lupus Erythematosus (SLE) is a disease of thousand faces whose manifestations vary with the interplay of genetic, ethnic, and host factors along with environmental exposures and geographical conditions. Since the first case of SLE reported in India in 1955, many case series have been published from various centers depicting Clinical and Immunological features in the Indian population. This study was undertaken to study the clinical and immunological profile in a tertiary care center in the sub-Himalayan region of northern India.
\end{abstract}

Materials and Methods: This study is a crosssectional retrospective study conducted at a tertiary care center in the hilly northern state of Himachal Pradesh over a period of 1 year; from 1st June 2017 to 31st May 2018. The records of all patients admitted in the study period with the department of medicine and diagnosed with SLICC 2012 classification criteria were studied to abstract the Clinical and Immunological profile of patients.

Results: A total of sixty-nine patients were included in the study. $96 \%$ of the subjects were female patients, and $84 \%$ of the patients were in the reproductive age group of 20-40 years. Mucocutaneous manifestations were most commonly seen in nearly $89 \%$ of patients followed by musculoskeletal involvement in about $71 \%$. Hematological involvement was more frequent than reported in the literature. Lupus Nephritis was diagnosed in about $30 \%$ of the patients. Fatigue and synovitis were the most common features, followed by oral ulcers and acute cutaneous lupus. ANA and ds DNA positivity was 98.5 and $72.4 \%$ respectively.

Six cases of MCTD were $d=$ found in the study group.

Conclusion: In hospitalized SLE patients admitted in a tertiary care center of the northern hilly state, females of reproductive age group constituted the majority of patients. Mucocutaneous, Musculoskeletal, hematological, and renal involvement were most prevalent.

Key Words: SLE, cross-sectional study, Mucocutaneous involvement, Renal Biopsy, Hilly area

\section{INTRODUCTION}

Systemic Lupus Erythematosus (SLE) is an enigmatic disorder aptly called "disease of thousand faces." It has varied clinical and laboratory manifestations with a plethora of autoantibodies. SLE has marked individual, ethnic, and geographical variations in the presentation and involvement of various organ systems. Outcomes also vary according to ethnicity and socioeconomic status.

The first case of SLE was reported in India in $1955 .^{1}$ Prevalence was said to be 
$3.2 / 100000$ of the population in rural area-based study done in $1993 .^{2}$ Indeed this prevalence is quite low if compared to the Indian migrants settled in other countries. While in epidemiological studies throughout the Asian continent, prevalence rates ranging from 4.3 to 43.5 per 100000 of the population have been reported. ${ }^{3}$ Given the variation in available information about the epidemiology of lupus in Asia, a concerted effort using standardized methods will be useful in providing clinically useful and relevant information about how the disease impacts in the region.

Various Indian studies have been published depicting the clinical and immunological profile of SLE patients in the Indian population. ${ }^{4,5,6,7,8,9}$ This study was undertaken to find the clinical and immunological profile of patients afflicted with SLE in the sub-Himalayan region of northern India at an altitude of 3000 meters above sea level.

\section{MATERIAL AND METHODS Setting:}

This study was conducted in Indira Gandhi Medical College, Shimla; a 700 bedded tertiary care center in the hilly state of Himachal Pradesh at an altitude of 3000 meters above sea level.

All the patients admitted with the diagnosis of SLE under the department of Medicine during the study period of $1^{\text {st }}$ June 2017 to $31^{\text {st }}$ May 2018 were included in the study.

\section{Data Collection}

The data were obtained retrospectively. A list of patients discharged with the diagnosis of SLE, as per SLICC classification criteria $2012,{ }^{10}$ during the study period was obtained from hospital records. All the data pertaining to the study was analyzed from the hospital records, including demographic, clinical, laboratory, and immunological parameters. Approval from the Institutional Ethics Committee was taken, and no personal data of patients were included in the study.

\section{Analysis}

Descriptive analysis of demographic, clinical, laboratory and immunological variables were done. The cumulative percentage frequency of selected variables was calculated using Epi Info software version 7.2.2.

\section{RESULTS}

Out of the Sixty-nine patients of SLE were included in the study, 95.65\%(66) were females. The mean age for the onset of SLE was 30.5 years. $84.06 \%$ of patients were in the reproductive age group of 20-40 years. Most prevalent clinical manifestations were fatigue (78.26\%), Oral Ulcers (72.46\%), Arthralgias (71.01\%), acute cutaneous rash $(56.52 \%)$ and photosensitivity (43.48\%). The most commonly involved system in the decreasing frequency was Mucocutaneous, Musculoskeletal, Haematological, and renal, with a cumulative percentage frequency of $89 \%, 71 \%, 65 \%$, and $37.6 \%$ respectively.

Table 1. Mucocutaneous manifestations

\begin{tabular}{|l|l|l|}
\hline \multicolumn{1}{|c|}{ Nable 1. Mucocutaneous manifestations } \\
\hline Acute Cutaneous Rash & 39 & $\%$ \\
\hline Chronic lupus & 6 & 56.52 \\
\hline Photosensitivity & 30 & 8.70 \\
\hline Oral Ulcers & 50 & 43.48 \\
\hline Alopecia & 27 & 72.46 \\
\hline Livedo reticularis & 1 & 39.13 \\
\hline
\end{tabular}

Table 2. Rheumatological manifestations

\begin{tabular}{|c|c|c|}
\hline & No. of patients & $\%$ \\
\hline Fatigue & 54 & 78.26 \\
\hline Synovitis & 49 & 71.01 \\
\hline Serositis & 11 & 15.94 \\
\hline Raynauds & 8 & 11.59 \\
\hline
\end{tabular}

Table 3 Haematological Manifestations.

\begin{tabular}{|l|l|l|}
\hline \multicolumn{2}{|c|}{ Table 3 Haematological Manifestations. } \\
\hline Anaemia & No. of patients & $\%$ \\
\hline Thrombocytopenia & 52 & 75.36 \\
\hline Leucopneia & 51 & 73.91 \\
\hline Autoimmune Anaemia & 34 & 49.28 \\
\hline
\end{tabular}

Table 4. Renal Abnormalities.

\begin{tabular}{|l|l|l|}
\hline & No. of patients & \% \\
\hline Urinary RBC & 19 & 27.54 \\
\hline Urinary CAST & 4 & 5.80 \\
\hline Albuminuria < 500 & 24 & 34.78 \\
\hline Albuminuria 30-500 & 21 & 30.43 \\
\hline No albuminuria & 24 & 34.78 \\
\hline Biopsy proven Lupus Nephritis & 21 & 30.43 \\
\hline
\end{tabular}

Table 5. Organ system Involvement

\begin{tabular}{|l|l|l|}
\hline & No. of patients & \% \\
\hline Gastrointestinal & 1 & 1.45 \\
\hline Cardiovascular & 2 & 2.90 \\
\hline Neurological & 6 & 8.70 \\
\hline Psychiatric & 2 & 2.90 \\
\hline Pulmonary & 7 & 10.14 \\
\hline Bad Obstetric History & 7 & 10.14 \\
\hline Family History & 4 & 5.80 \\
\hline
\end{tabular}

In mucocutaneous system, Oral Ulcers were most frequent $(72.46 \%)$ followed by Acute Cutaneous rash (56.5\%), Photosensitivity (43.48\%), Alopecia (39.13\%) and Chronic Lupus (8.7\%). Raynaud's phenomenon was present in $11.59 \%$ of patients. In musculoskeletal system, joint involvement was 
seen in $71.01 \%$ of patients. In haematological system, Anaemia was the most common form of involvement seen in $75.6 \%$ patients followed by thrombocytopenia $(73.9 \%)$ and leucopenia (49.2\%). Most common renal involvement was in the form of Albuminuria (> 500mg/24hrs) in $34.78 \%$ of patients. $27.5 \%$ of patients had urinary RBC's while $5.8 \%$ had Urinary Casts. $30.43 \%$ were diagnosed with Lupus Nephritis on Renal Biopsy.10.14\% of patients had pulmonary involvement in the form of Interstitial Lung Disease and Pulmonary Arterial Hypertension. Frequency of various sign symptoms and involvement of organ systems is tabulated in Table 1- 6.

Table 6. Autoimmune Profile of study participants.

\begin{tabular}{|l|l|l|}
\hline Auto-antibody & No. of patients & $\%$ \\
\hline ANA & 68 & 98.55 \\
\hline ds DNA & 50 & 72.46 \\
\hline Anti Sm & 14 & 20.29 \\
\hline Anti Ro/La & 36 & 52.7 \\
\hline U1 RNP & 12 & 17.39 \\
\hline Anti histone & 12 & 17.39 \\
\hline Anti centromere & 7 & 10.14 \\
\hline scl 70 & 5 & 7.25 \\
\hline APLA & 3 & 4.35 \\
\hline C3,C4 low & 36 & 52.17 \\
\hline DCT & 27 & 39.13 \\
\hline
\end{tabular}

Table 7. Comparison of study findings with other studies.

\begin{tabular}{|c|c|c|c|c|c|c|c|}
\hline $\begin{array}{l}\text { Clinical /immunological } \\
\text { Manifestations }\end{array}$ & $\begin{array}{l}\text { Malviya }^{4} \\
(1988) \\
n=329 \\
(\%)\end{array}$ & $\begin{array}{l}\text { Malviya }^{5}(1997) \\
\mathrm{n}=1366(\%)\end{array}$ & $\begin{array}{l}\text { Binoy }^{6} \\
(2003) \\
n=75 \\
(\%) \\
\end{array}$ & $\begin{array}{l}\text { Kosaraju }^{7}(2010) \\
\mathrm{n}=48(\%)\end{array}$ & $\begin{array}{l}\text { Saigal }^{8} \\
(2011) \\
\mathrm{n}=60 \\
(\%) \\
\end{array}$ & $\begin{array}{l}\text { Agrawal }^{9}(2013) \\
\text { n }=87(\%)\end{array}$ & $\begin{array}{l}\text { Present } \\
\text { study } \\
n=69 \\
(\%) \\
\end{array}$ \\
\hline Malar rash & 85 & 58.5 & 40 & 35.41 & 43.3 & 71.3 & 56.52 \\
\hline Discoid rash & NA & 7 & 5.3 & NA & 1.7 & 32.2 & 8.7 \\
\hline Alopecia & 82 & NA & 60 & 18.75 & 65 & 10.34 & 39.13 \\
\hline Oral Ulcer & 64 & 57 & 64 & 25 & 61.7 & 42.35 & 72.46 \\
\hline Photosensitivity & 67 & 57 & 64 & 25 & 61.7 & 42.53 & 69 \\
\hline Fatigue & NA & NA & NA & NA & NA & NA & 78.26 \\
\hline Synovitis & 92 & 85 & 89.3 & 64.58 & 86.7 & 52.9 & 71.01 \\
\hline Raynauds & NA & NA & NA & NA & NA & NA & 11.59 \\
\hline Hemolytic Anaemia & 7 & 4 & 1.3 & 2 & 25 & 8.1 & 39 \\
\hline Leucopenia & 16 & NA & 14.7 & NA & 43.3 & 18.4 & 49.28 \\
\hline Thrombocytopenia & 11 & 9 & 12 & NA & 33.3 & 14.9 & 73.19 \\
\hline Renal & 73 & 57 & 33.3 & 20.83 & 56.7 & 69 & $37.6 \%$ \\
\hline Lupus Nephritis & NA & NA & NA & NA & NA & NA & $30.43 \%$ \\
\hline CVS & 29 & NA & 5.3 & NA & 6.7 & 2.3 & 2.9 \\
\hline GIT & NA & NA & NA & NA & NA & NA & 1.43 \\
\hline Pulmonary & NA & NA & 8 & 12.5 & 11.7 & 12.6 & 10.14 \\
\hline Neuropsychiatric & 38 & 51 & 13.3 & 8.33 & 13.3 & 4.6 & 11.1 \\
\hline ANA & 98 & 97 & 93.3 & 64.28 & 98.3 & 97.7 & 98.55 \\
\hline ds DNA & 55 & 68 & 76 & 89.36 & 65 & 93.9 & 72.46 \\
\hline ANti Sm & NA & NA & NA & NA & NA & NA & 20.29 \\
\hline Anti Ro/La & NA & NA & NA & NA & NA & NA & 52.17 \\
\hline U1 RNP & NA & NA & NA & NA & NA & NA & 17.39 \\
\hline APLA & NA & NA & NA & NA & NA & NA & 4.35 \\
\hline No. of MCTD cases & NA & NA & NA & NA & NA & NA & 8.7 \\
\hline No. of deaths & NA & NA & NA & NA & NA & NA & 5.79 \\
\hline
\end{tabular}

In our study we found one case each of Lupus Enteritis, Acute Lupus Pneumonitis, Conduction heart block and Libmann sack's Endocarditis. One patient was diagnosed with Candidal knee joint arthritis as complication of prolonged immunosuppression. ANA positivity was found in 68 patients (98.55\%). One patient was diagnosed with ANA negative SLE on the basis of biopsy-proven Lupus Nephritis. Ds DNA antibodies were seen in 50 patients (72.46 $\%$ ), followed by Anti Ro/La antibodies in 26 patients (37.68\%). APLA were found in 3 patients $(4.35 \%)$. Four patients of SLE died in our hospital during the study period. Two of these patients succumbed to Sepsis after receiving Immunosuppression for Lupus Nephritis. The other two deaths were attributed to Severe PAH leading to RV dysfunction and Cardiogenic shock and Acute lupus Pneumonitis.

\section{DISCUSSION}

To the best of our knowledge, this is the first study on SLE from India representing the sub-Himalayan northern Indian population, approximately 3000 meters above the sea level.

In our study, $95 \%$ of the patients were female. In various Indian studies this ranges from $84-95 \% .^{4,5,6,8,9,10}$ Mean age at onset in our study is 30.5 years which is also consistent with other India studies. We had two male patients out of 69 patients. One presented with Synovitis and pericarditis and the other one presented with predominant renal involvement and diagnosed 
with Lupus Nephritis. Cumulative Incidence of Mucocutaneous manifestations was $89 \%$ in our study, with $62 \%$ having cutaneous manifestations in the form of Acute, subacute, or chronic lupus manifestations. $69 \%$ of patients with cutaneous involvement had photosensitivity. Dermatological participation in various studies from India varies from $64-85 \%$. $4,5,6,7,8,9$

Similarly, frequency of acute cutaneous lupus has been ranged from $40-85 \%$ in various studies and in the present study we found Acute cutaneous involvement in $56.52 \%$ of patients and Chronic Lupus in $8.7 \%$, while chronic lupus has been found to vary from $1-7 \%$ in various studies. ${ }^{4,5,6,7,8,9}$ Photosensitivity has been reported in the range of $27-75 \%$ in Indian studies and our study photosensitivity was found in $69 \%$ of patients afflicted with cutaneous manifestations. Except in one study from Western India (Saigal et $\mathrm{al}^{8}$ ), Haematological Involvement is seen less frequently in other Indian studies as compared to our study. We had one case of ANA negative SLE proven with renal biopsy and thus ANA positivity rate of 98 . $5 \%$ with ds DNA and Anti Sm positivity of $72.48 \%$ and $20.29 \%$ consistent with literature.

We had anti-Ro/La antibodies positive in 36 patients. Table 7 .

Only three patients with Anti Ro/La positive antibodies were found to have Lupus nephritis, and 22 patients had photosensitivity. This too consistent with the association of Anti $\mathrm{Ro} / \mathrm{La}$ antibodies, as mentioned in literature. Our study had a few limitations. It was a single retrospective Centre hospital-based study, with a lack of adequate follow-up. Patients with lesser severity of the illness being treated on an outpatient basis have not been represented in this study. A prospective multicenter study is required to confirm the findings of our study.

\section{CONCLUSION}

This study shows a high prevalence of Mucocutaneous and Musculoskeletal features in SLE patients consistent with the literature. Our study has higher hematological involvement as compared to other Indian studies. The immunological profile of patients in this study was consistent with that mentioned in the literature. Few rare presentations like Lupus Enteritis, Conduction Heart block, Acute Lupus Pneumonitis were encountered during this study. Ours is probably the first study to document a subset of MCTD cases in SLE patients. Still, our study doesn't evaluate a representative sample, and thus extensive multicenter and community-based studies are required for accurate depiction of SLE manifestation in our country. Further, Rheumatological disease registries should be set up in Tertiary health care centers for proper follow up and reporting of SLE cases.

\section{Acknowledgement: None}

\section{Conflict of Interest: None}

\section{Source of Funding: None}

\section{Ethical Approval: Approved}

\section{REFERENCES}

1. Desai SC, Bhandarkar SD. A case report of acute disseminated lupus erythematosus. Ind J Dermatol V1e9ne5r5io;1 21: 131-134.

2. Malviya A N et al. Prevalence of systemic lupus erythematosus in India. Lupus. 1993 Apr;2(2):115-8.

3. E Osio-Salido and $\mathrm{H}$ Manapat-Reyes Epidemiology of systemic lupus erythematosus in Asia. Lupus (2010) 19, 1365-1373.

4. Malaviya AN, Singh RR, Kumar A, De A, Kumar A, Aradhye S. Systemic lupus erythematosus in northern India: A review of 329 cases. J Assoc Physicians India 1988;36:476-80, 484.

5. Malaviya AN, Chandrasekaran AN, Kumar A, Shamar PN. Systemic lupus erythematosus in India. Lupus 1997;6:69070

6. Binoy J Paul et al. Clinical Profile of SLE in Northern Kerala.J Indian Rheumatol Assoc $2003: 11 ; 94$ - 97.

7. Kosaraju K, Shenoy S, Suchithra U. A cross-sectional hospital-based study of autoantibody profile and clinical manifestations of systemic lupus erythematosus in south Indian patients. Indian J Med Microbiol 2010;28:245-7.

8. Saigal R, Kansal A, Mittal M, Singh Y, Maharia HR, Juneja M. Clinical profile of systemic lupus erythematosus patients at a tertiary care centre in Western India. J Indian Acad Clin Med 2011;13:27-32.

9. Agrawal SR, Tiewsoh I, Rajput A, Jain A. A cross-sectional hospital based study of clinical and immunological profile of 
Varun Jaswal et.al. A cross-sectional study of clinical and immunological profile of SLE patients from a tertiary care center in the Sub Himalayan region of Northern India.

Systemic lupus Erythematosus patients from central rural India. Indian J Allergy Asthma Immunol.2013;27:33-7.

10. Petri, M. , Orbai, A. , Alarcón, G. S., Gordon, C. , Merrill, J. T., Fortin, P. R., et al Derivation and validation of the Systemic Lupus International Collaborating Clinics classification criteria for systemic lupus erythematosus. Arthritis \& Rheumatism. 2012; 64: 2677-2686.
How to cite this article: Jaswal V, Mokta JK, Chauhan V et.al. A cross-sectional study of clinical and immunological profile of SLE patients from a tertiary care center in the Sub Himalayan region of Northern India. Gal Int $J$ Health Sci Res. 2021; 6(3): 57-61. DOI: https:// doi.org/10.52403/gijhsr.20210709 